\title{
ASIA syndrome, calcinosis cutis and chronic kidney disease following silicone injections. A case-based review
}

\author{
Giuseppe Barilaro ${ }^{1}$ (1) Claudia Spaziani Testa ${ }^{1} \cdot$ Antonella Cacciani $^{1} \cdot$ \\ Giuseppe Donato $^{1} \cdot$ Mira Dimko $^{2} \cdot$ Amalia Mariotti $^{2}$
}

Published online: 24 September 2016

(c) Springer Science+Business Media New York 2016

\begin{abstract}
An immunologic adjuvant is a substance that enhances the antigen-specific immune response preferably without triggering one on its own. Silicone, a synthetic polymer used for reconstructive and cosmetic purposes, can cause, once injected, local and/or systemic reactions and trigger manifestations of autoimmunity, occasionally leading to an overt autoimmune disease. Siliconosis, calcinosis cutis with hypercalcemia and chronic kidney disease have all been reported in association with silicone injection. Here, we describe a case of autoimmune/autoinflammatory syndrome induced by adjuvants, calcinosis cutis and chronic kidney disease after liquid silicone multiple injections in a young man who underwent a sex reassignment surgery, followed by a review of the literature. To our knowledge, this is the first report describing the concomitance of the three clinical conditions in the same patients. The link between silicone and the immune system is not completely understood yet and requires further reports and investigations with long-term data, in order to identify the main individual and genetical risk factors predisposing to the wide spectrum of the adjuvant-induced responses.
\end{abstract}

Keywords ASIA syndrome $\cdot$ Siliconosis $\cdot$ Calcinosis cutis $\cdot$ Hypercalcemia $\cdot$ Chronic kidney disease

Giuseppe Barilaro

giuseppe.barilaro@uniroma1.it

1 Clinical Immunology Unit, Department of Clinical Medicine, Sapienza University of Rome, Viale dell'Università 37, 00161 Rome, Italy

2 Nephrology Unit, Department of Clinical Medicine, Sapienza University of Rome, Viale dell'Università 37, 00161 Rome, Italy

\section{Introduction}

Immune-mediated diseases are caused by a complex of genetic and environmental factors. A genetically susceptible subject may develop an autoimmune or inflammatory disease following the exposure to a specific external factor. An immunologic adjuvant (word derived from latin adjuvare, which means help) is a substance that enhances the antigen-specific immune response, normally without triggering one on its own. Many substances are used in medicine as adjuvants, mainly to boost the immune response to vaccination. These components increase the local reaction to antigens and subsequently the release of chemokines and cytokines from T-helper and mast cells. An adjuvant, while inducing the immune system activation, a desirable effect, can trigger manifestations of autoimmunity, sometimes leading to an overt autoimmune disease.

In recent years, four medical conditions, namely siliconosis, the Gulf War syndrome (GWS), the macrophagic myofasciitis syndrome (MMF) and postvaccination phenomena, were linked with previous exposure to an adjuvant substance. Since these four entities share a similar complex of symptoms and signs and a common pathogenesis, Shoenfeld and Agmon-Levin suggested to gather them together under a common syndrome denominated 'Autoimmune/auto-inflammatory Syndrome Induced by Adjuvants' [1] (ASIA), and proposed a set of diagnostic criteria for this entity (Table 1) [2].

ASIA syndrome can be considered a multifactorial process triggered by the exposure to certain environmental factors, such as infections, toxins, drugs, vaccine adjuvants, biomaterials and stress. Main substances associated with the onset of ASIA syndrome are squalene (Gulf War syndrome), aluminum hydroxide (postvaccination phenomena, macrophagic myofasciitis syndrome) and silicone 
Table 1 Suggested criteria for the diagnosis of 'ASIA' (for positive diagnosis of ASIA, fulfilment of either two major or one major and two minor criteria is required)

Major criteria:

Exposure to an external stimuli (infection, vaccine, silicone, adjuvant) prior to clinical manifestations

The appearance of 'typical' clinical manifestations:

Myalgia, Myositis or muscle weakness

Arthralgia and/or arthritis

Chronic fatigue, un-refreshing sleep or sleep disturbances

Neurological manifestations (especially associated with demyelination)

Cognitive impairment, memory loss

Pyrexia, dry mouth

Removal of inciting agent induces improvement

Typical biopsy of involved organs

Minor criteria:

The appearance of autoantibodies or antibodies directed against the suspected adjuvant

Other clinical manifestations (e.g. irritable bowel syndrome)

Specific HLA (i.e. HLA DRB1, HLA DQB1)

Onset of an autoimmune disease (e.g. multiple sclerosis, systemic sclerosis)

ASIA autoimmune/auto-inflammatory syndrome induced by adjuvants

(siliconosis), while mineral oils, guaiacol and iodine gadital are less common triggers [3, 4].

Silicone is a synthetic polymer used for reconstructive and cosmetic purposes. Although it used to be thought of as an inert material, uncapable to provoke immune activation, soon after its introduction, it has been associated to the onset of autoimmune/inflammatory manifestations. In addition, there are reports in the literature describing the onset of calcinosis cutis, hypercalcemia and chronic kidney disease in patients who underwent silicone injections [5]. In this article, we report a case of siliconosis, calcinosis cutis with hypercalcemia and chronic kidney disease after multiple injections of liquid silicone, followed by a review of the literature.

\section{Materials and methods}

We performed a literature search for articles published from 1964 to August 2016. We used the MEDLINE database (PubMed, National Library of Medicine, Bethesda, MD). We combined the following main keywords: ASIA syndrome, siliconosis, hypercalcemia, chronic kidney disease and calcinosis cutis. The reference lists of all articles were scanned for references not identified in the initial research.

\section{Case report}

A 48-year-old Hispanic woman presented to our observation with fever, fatigue, nausea, vomiting, abdominal pain and diffuse arthralgias. Her clinical history was remarkable for a male-to-female sex reassignment surgery along with mastoplasty and multiple injections of liquid silicone, chronic renal failure, arterial hypertension and dyslipidemia.

She was pretty dehydrated, while the rest of physical examination was unremarkable except for the presence of extensive, palpable, non-tender, subcutaneous calcifications on the lower abdomen and gluteus likely related to the previous silicone injections. Moreover, she presented ulcers on both buttocks, in the site of previous silicone injection, both surrounded by skin hyperpigmentation, the left one being likely infected.

Laboratory tests showed leukocytosis, increased inflammatory indices, relevant increase of serum creatinine (6.4 mg/dl with normal values $<1.0 \mathrm{mg} / \mathrm{dl}$ ), hypercalcemia $(14.7 \mathrm{mg} / \mathrm{dl}$ with n.v. $<11 \mathrm{mg} / \mathrm{dl})$ and hypercalciuria (397 mg/24 h), increased alkaline phosphatase (ALP) (151 UI/L n.n. $<50 \mathrm{UI} / \mathrm{L})$, but low serum intact parathyroid hormone levels $(5.3 \mathrm{pg} / \mathrm{ml})$ and normal 1,25 dihydroxyvitamin D levels. HIV serology was negative. Serum immunoelectrophoresis and Bence Jones protein research were both negative, thus excluding a multiple myeloma. Primary hyperparathyroidism was excluded because of low serum intact parathyroid hormone levels $(5.3 \mathrm{pg} / \mathrm{ml})$. Other possible causes of hypercalcemia such as paraneoplastic syndrome, milk-alkali syndrome, sarcoidosis or excessive use of vitamin D were ruled out. Tuberculosis was excluded as well (quantiferon ${ }^{\circledR}$-TB Gold In-Tube Test negative). Laboratory workup also included antinuclear antibodies (ANA), rheumatoid factor (RF), antineutrophil cytoplasmic antibodies (ANCA) and anti-extractable nuclear antigens (ENA) testing that were all negative. 
The patient underwent a magnetic resonance imaging (MRI) of the abdomen which showed numerous subcutaneous areas, hyper-intense on T2-weighted sequences, classifiable as siliconomas (Figs. 1, 2).

A renal biopsy showed severe and diffuse sclerosis, basal membranes with variable thickness, neutrophil infiltration of vessel lumen and hyperplasia of juxtaglomerular cells. In particular, sclerotic glomerula presented collapsed basal membranes and deposition of amorphous undercapsular collagen. Direct immunofluorescence showed deposition of $\operatorname{IgM}$ and $\mathrm{C} 3$ in vessel walls and in sclerotic zones. The ultrastructural examination showed an increase of mesangial matrix, extended interstitial fibrosis, severe tubular damage with tubular basal membranes thickened and rich of granular material.

A diagnosis of ASIA syndrome was made as the patient fulfilled three of the four major criteria proposed by Shoenfeld and Agmon-Levin. Prednisone $(0.5 \mathrm{mg} / \mathrm{Kg})$ with massive intravenous hydration and furosemide were started, with progressive renal function recovery and normalization of calcium levels. A gluteus wound swab showed the presence of Escherichia coli, so a prompt antibiotic therapy with ertapenem was administered, with progressive recovery of the infected lesions. Needless to say, surgical removal of all scattered lumps was not a viable option because of the excessive and diffusely infiltrating presence of silicone all over the body.

\section{Discussion}

An immunologic adjuvant is a substance that accelerates and enhances the antigen-specific immune response. Adjuvants may act in several ways: by increasing the local reactions in the site of injection by mimicking danger signals [6-8]; by inducing a progressive release of the antigen, hindering its clearance and enabling a longer exposure of the antigen to antigen-presenting cells; by

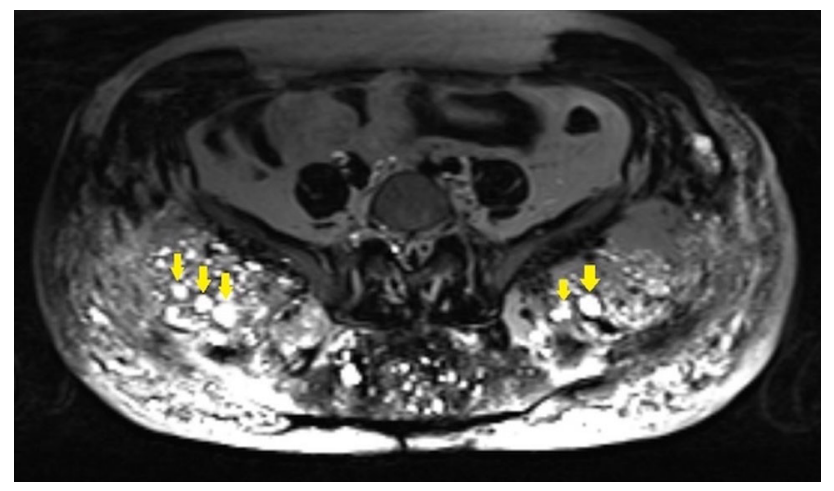

Fig. 1 Hyper-intense areas on T2-weighted sequences (indicated by arrows) represent siliconomas of the buttocks

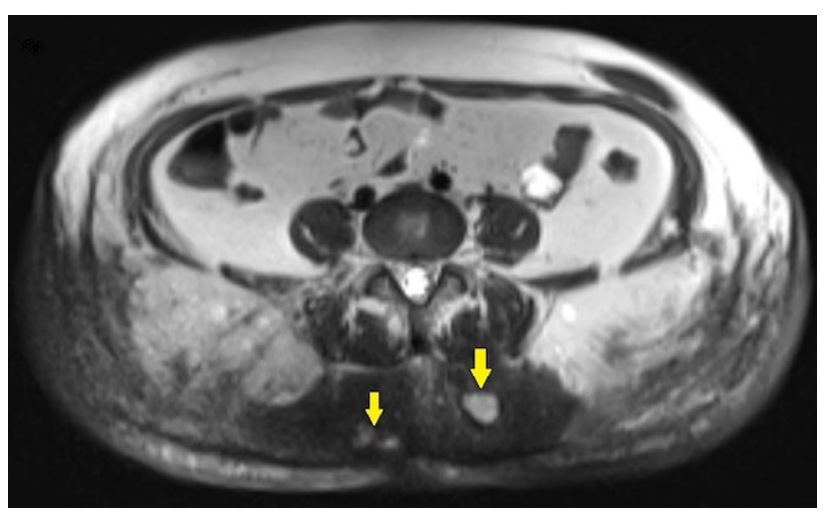

Fig. 2 Hyper-intense paravertebral siliconomas on T2-weighted sequences (indicated by arrows)

promoting the translocation of antigens to the lymph nodes where antigens can be recognized by $\mathrm{T}$ cells; by inducing the release of inflammatory cytokines $[9,10]$; by interacting with toll-like receptors (TLRs) and nucleotide oligomerization domain (NOD)-like receptors (NLRs) such as NALP3 inflammasome [11]. The activation of the immune system triggered by these substances is normally self-limited. However, in genetically susceptible subjects, it can be out of control triggering the production of autoantibodies, and occasionally, an overt autoimmune disease.

Silicones are a family of synthetic polymers sharing a silicone-oxygen chain with varying organic side groups. Since their introduction, they were incorporated into a myriad of medical products and devices such as breast and joint implants, ventriculoperitoneal shunts, artificial heart valves, intraocular lenses and more [12]. The three common forms of silicone are elastomer (silastic), liquid and gel. At present, the most commonly used form is gel, which was first introduced in 1960s for reconstructive and cosmetic purposes as a component of breast implants (SBI). Liquid silicone was used for breast augmentation in the 1950 and 1960s until reports of adverse effects appeared in the United States. Initially thought of as inert material, it was discovered that liquid silicone can invade deep muscle tissue and trigger fibrosis, granuloma formation and lymphadenopathy. It can migrate through tissue planes to dependent areas such as the lower abdominal wall, the inguinal canal and the buttocks [13]. Occasionally, it can reach the central nervous system through a transfer operated by macrophages as has been seen for alum [14]. Fibrosis is less effective in barricading a liquid irritant than a solid one; therefore, these masses are able to grow to large sizes.

Injection of silicone can trigger both local and systemic reactions. Following silicone implantation, a capsule forms around the implant as part of an inflammatory response to a foreign body [15]. In particular, postoperative wound 
healing is characterized by acute inflammation followed by the formation of a myofibroblast-containing fibrotic capsular tissue with CD4 + lymphocytes, macrophages and multinucleated giant cells that surround the implant, known as 'Siliconoma' [16]. The appearance of capsular fibrosis around the prosthesis is very frequent, but the mechanism which underlies this phenomenon has not been fully understood yet. Recently, it has been proposed that silicone implants trigger a specific antigen-driven local immune response through activated Th1/Th17 cells, suggesting that fibrosis is promoted by the production of profibrotic cytokines as a consequence of faltering function of local $\mathrm{T}$ regulatory cells [17].

Once injected, silicone is able to stimulate the activation of local macrophages, which results in cytokines and reactive oxygen species production. The process of oxidation leads to macrophage apoptosis resulting in the release of silicone containing particles that can be taken up once again by other macrophages, amplifying the inflammatory process [18]. After breast reconstruction with prostheses containing silicone gel, implant rupture, silicone leakage through the shell, elastomer fragmentation, capsular fibrosis and capsular contracture, silicone granuloma, local inflammation and regional lymphadenopathy have been reported $[19,20]$. In addition, patients with immunemediated reactions to implanted silicone were found to have increased $\mathrm{IgG}$ in the surrounding tissue and higher levels of anti-silicone antibodies compared with asymptomatic implanted patients [21]. A remission of such clinical features following removal of silicone gel breast implants has been described as well [22, 23]. Another local side effect to implants can be an allergic reaction to either silicone or platinum, a catalyst used in silicone polymerization found in minute quantities [24]. Even in the absence of implant rupture, diffusion of silicone through the envelope into the surrounding tissues, called 'bleeding', may represent a complication [25]. Bleeding increases with time and enhances the inflammatory response around the capsule.

Immune-mediated phenomena and autoimmune diseases following exposure to silicone have been an issue of debate for many years. Silicone was previously considered to be an inert material, but like other adjuvants, it is able to induce autoimmune-like phenomena termed in the early 1990s 'Human adjuvant disease' [26]. The association between silicone implants and the onset of well-defined autoimmune diseases have been postulated by several authors [27], but it still remains controversial. Systemic adverse reactions to silicone implants have been reported since 1964 when Miyoshi et al. [28] described the case of two patients who developed a connective tissue disease several years following a breast implant augmentation and coined the acronym of "human adjuvant disease" (HAD).
Thenceforth silicone exposure has been linked to several autoimmune/rheumatic diseases such as multiple sclerosis [29] or chronic fatigue syndrome [30]. Case reports and case series of rheumatoid arthritis, Sjögren's syndrome, systemic lupus erythematosus, mixed connective tissue disease as well as other autoimmune diseases developing in women who underwent silicone breast implants (SBI) appeared in the medical literature soon after their introduction, suggesting a causal relationship between breast implants and the onset of an autoimmune disease [31-33]. Cases of scleroderma following silicone breast implants have been reported [34]. Lofgren syndrome and neurosarcoidosis have been described as late complications of augmentation mammoplasty using silicone gel. Remission following removal of silicone gel breast implants has been reported in a patient with debilitating multisystem sarcoidosis [23, 35]. In a large cohort study published by Hennekens and colleagues, based on self-reported symptoms of 10,830 implanted women, the authors found a relative risk of 1.25 (95\% CI: 1.08-1.41) for implanted women compared to non-implanted women for all defined connective tissue diseases [36]. Nevertheless, a metaanalysis published by Janowsky et al. [37] that did not include the former study concluded that there was no increased risk of defined connective tissue diseases following silicone breast implantation (RR 0.80; $95 \% \mathrm{CI}$ : 0.62-1.04). Therefore, since different studies reached divergent conclusions about the link between silicone breast implants and defined autoimmune diseases, this association remains controversial [38, 39].

The finding of different autoantibodies in patients who underwent silicone breast implants (SBI) [40] is not uncommon. Zandman-Goddard et al. [41] have shown that the presence of autoantibodies is increased in symptomatic silicone breast implants (SBI)-implanted women compared to asymptomatic women who had undergone the procedure.

On the other hand, the relationship between silicone implants and a particular constellation of symptoms, which did not fulfill the diagnostic criteria for any recognized connective tissue disease, has been documented in several studies. Fryzek et al. [42] showed that a group of 1546 patients with silicone breast implants had a statistically significant increase in 16 of 28 investigated symptoms when compared to a group of 2496 women who underwent reduction mammoplasties. These control patients, having had cosmetic breast surgery, attenuated the possibility of confounding factors. Many of these symptoms satisfied several criteria for fibromyalgia and chronic fatigue syndrome. Vera-Lastra et al. [43] recently published a case series of 50 patients with ASIA syndrome after illegal injection of foreign substances. Among all patients, thirty $(60 \%)$ presented with non-specific manifestations of 
autoimmune rheumatic diseases and twenty (40\%) fulfilled the criteria for a defined autoimmune disease. All patients were treated with corticosteroids and had clinical improvement. A surgical removal of the foreign material was performed whenever possible, and most patients showed clinical improvement. Vasey et al. [44] found a statistically significant correlation between silicone breast implants and the presentation of many aspecific signs and symptoms such as body ache, joints pain, myalgia, fatigue, impaired cognition and others. Cohen Tervaert et al. [18] published an interesting study in which they evaluated 32 patients who formerly underwent silicone-filled breast implants surgery, showing symptoms suggestive for incompatibility with the implants. These patients were evaluated for ASIA syndrome, immunodeficiency and autoimmune diseases. Seventeen over 32 patients were diagnosed with a systemic autoimmune disease, and 15 of them showed impaired humoral immune system. All patients suffered from an array of signs and symptoms and fulfilled the diagnostic criteria for ASIA. Median time between start of complaints and prosthesis implantation was 10 years (2-24 years). Patients with enlarged lymph nodes underwent a biopsy, and the most common histological finding was a foreign body reaction with granulomatous inflammation containing multiple giant cells suggestive of biopsy-proven siliconosis. Therefore, the exposure to silicone in genetically prone subjects can trigger the development of an array of symptoms and signs such as body ache, abnormal fatigue, impaired cognition, depression, dry eyes, dry mouth, skin abnormalities, paresthesia, swollen and tender axillary glands, unexplained fever, hair loss, headache and morning stiffness that can be referred to as 'siliconosis'. Hence, the link between silicone and autoimmunity should not be limited by the constraints of defined diseases, but rather correlate to the relatively prevalent non-defined symptoms.

In order for silicone to act as an environmental trigger, there has to be a genetic predisposition for developing such clinical conditions [45]. The relationship between genetic susceptibility and environmental factors has gained increased attention and is discussed extensively in recent literature, including discussions of geoepidemiology, epigenetics, infection, animal models, use of twins and gender [46]. The case of two HLA-matched sisters who both received silicone breast implants and developed poly-articular arthritis and neurological symptoms has been described [47]. Although silicone implants are safe for the vast majority of subjects, screening for autoimmune phenomena and genetic testing can be useful tools for future risk stratification before implantation to help avoid adverse events in predisposed subjects [48].

Current generation implants are designed with a thicker shell, more cohesive gel and a barrier coating on the inner surface of the outer shell to decrease silicone leakage. However, Colaris et al. [49] recently published the results of a study in which they compared the clinical manifestations of two cohorts (one hundred patients each) with ASIA syndrome secondary to silicone breast implants, the first one diagnosed in Mastricht in 2014 and the second in Baylor College of Medicine (Houston) between 1985 and 1992. The authors did not find substantial differences in terms of clinical manifestations between the two cohorts; therefore, they concluded that despite changes in the principal constituents of the silicone implants during the past 50 years, silicone remained an adjuvant that may 'bleed' and subsequently may be a chronic stimulus to the immune system resulting in similar clinical manifestations.

Calcinosis cutis is a group of disorders initially described by Virchow in 1855, in which calcium is deposited in the skin. Calcium phosphate deposition occurs in response to local or systemic factors that drive the concentration above its solubility product [5]. Dystrophic calcinosis occurs in a normal metabolic state when tissue has been damaged or devitalized by some pathologic process. It is thought that damaged tissue exposes denatured proteins which bind to free phosphate, and hence, the calcium reacts with bound phosphate to precipitate calcium phosphate crystals. These crystals in turn increase the tissue damage, propagating the process and leading to the formation of giant accretions. These accretions are often the focus of intense granulomatous fibrosis which limits their growth [50]. Calcinosis cutis occurs most often in association with chronic kidney disease, cancer, [51] and autoimmune conditions such as CREST syndrome (CC, Raynaud's phenomenon, esophageal dysfunction, sclerodactyly and telangiectasia) [52].

Hypercalcemia in association with granulomatous diseases (especially sarcoidosis) has been extensively described in the literature. It has been related to a dysregulated activity of $1 \alpha$-hydroxylase enzyme $(1 \alpha \mathrm{HE})$ in giant cells and macrophages from granulomas that leads to an uncontrolled production of 1,25-dihydroxycholecalciferol $(1,25 \mathrm{D} 3)$ in calcified granulomas [53]. Macrophage $1 \alpha$ hydroxylase enzyme is completely unregulated by feedback mechanisms unlike renal $1 \alpha$-hydroxylase enzyme [54]. Corticosteroids are valuable in all forms of hypercalcemia as they reduce gastrointestinal calcium absorption and inhibit osteoclast function. But, they are particularly effective in granulomatous diseases as sarcoidosis because of their effects on vitamin D metabolism. Even though they have no effect on 1a-hydroxylase of renal tubular cells, they are potent inhibitors of macrophage $1 \alpha$-hydroxylase enzyme [55]. An alternative explanation for granulomatous diseases-associated hypercalcemia is the overproduction of PTH-related proteins (PTHrP). Increased serum levels of PTHrP have been documented in sarcoidosis along with 
immunohistochemical staining for PTHrP of macrophages and multinucleated giant cells within the granulomatous tissue [56]. Corticosteroids bind to cytokine promoter regions in the macrophage nucleus down-regulating IL-6 and IFN-gamma expression, and ultimately leading to a reduced PTHrP production by macrophages [57].

The association between silicone injection, granulomas and hypercalcemia has been reported since 1984 [58], and there are several reports in the literature [59-62]. As for the other granulomatous diseases, hypercalcemia is likely the consequence of a dysregulated production of 1,25-dihydroxivitamin $\mathrm{D}$ by activated macrophages (induced by tumor necrosis factor- $\alpha$ and interferon- $\gamma$, and inhibited by corticosteroids) [63] and/or an increase of PTHrP levels (stimulated by TNF- $\alpha$ and IL-6) [64]. This hypothesis is supported by the excellent response of hypercalcemia to glucocorticoid therapy which decreases PTHrP levels and inhibits the macrophage-derived 1 - $\alpha$-hydroxilase leading to a 1,25-dihydroxivitamin $\mathrm{D}$ reduction. Minocycline, cyclosporine and allopurinol have been used with some success as well [65]. Of note, hydroxychloroquine and ketoconazole have been shown to be effective in treating hypercalcemia in sarcoidosis [66]. In contrast to long-term steroids, their milder side effect profiles might make them preferable candidates, but their exact role in treating siliconomas and paraffinomas is still to be explored. Further studies are necessary to better understand the pathophysiological mechanisms of silicone-induced hypercalcemia and establish the best treatment options.

Renal failure has been extensively described in granulomatous diseases, such as sarcoidosis. The main pathophysiological mechanism of kidney damage seems to be a granulomatous inflammation confined to the tubulointerstitial compartment [67], but interstitial and membranous nephritis without any evidence of granuloma has been reported as well [68]. Nevertheless, the exact mechanism of development of renal failure in siliconomas- or paraffinomas-associated granulomatous disease is still unclear and further reports are needed.

The peculiarity of our case is represented by the complex clinical picture with the coexistence of the three main clinical manifestations related to silicone injections being the autoimmune/inflammatory phenomena, calcinosis cutis with hypercalcemia, and chronic kidney disease. To our knowledge, this is the first report describing the concomitance of the three conditions in the same patients. As we observed in our patient, when the surgical removal of siliconomas is not possible, corticosteroids represent an excellent treatment option with a very good overall clinical response.

Most of the reports available in the literature suggest that silicone implants cause a negligible and non-specific foreign body reaction. Occasionally, they can trigger autoantibodies production and autoimmune diseases, but they are more likely associated with an array of non-defined rheumatic symptoms. Nevertheless, as analyzed by Jara et al. [69] in a recent systematic review regarding severe manifestations of ASIA syndrome, even though the percentage of severe cases is small (about $7 \%$ of all cases of ASIA syndrome), this condition can be life-threatening.

The relationship between silicone and the immune system requires further reports and investigations with longterm data in order to identify the main individual and genetic risk factors predisposing to the wide spectrum of the adjuvant-induced responses.

\section{Conclusion}

Silicone injections can be very harmful, triggering in the same patient the onset of ASIA syndrome, calcinosis cutis, hypercalcemia, and chronic kidney disease. In the event of such life-threatening manifestations, surgical removal of prostheses should be mandatory. When eliminating the trigger is not possible, as in our patient, corticosteroids are the best and most effective therapy.

Acknowledgments This research received no specific grant from any agency in the public, commercial or not-for-profit sectors.

Compliance with ethical standards

Conflict of interest None declared.

\section{References}

1. Shoenfeld Y, Agmon-Levin N. 'ASIA'-autoimmune/inflammatory syndrome induced by adjuvants. J Autoimmun. 2011; 36(1):4-8. doi:10.1016/j.jaut.2010.07.003.

2. Meroni PL. Autoimmune or auto-inflammatory syndrome induced by adjuvants (ASIA): old truths and a new syndrome? J Autoimmun. 2011;36(1):1-3. doi:10.1016/j.jaut.2010.10.004.

3. Vera-Lastra O, Medina G, Cruz-Dominguez Mdel P, Jara LJ, Shoenfeld Y. Autoimmune/inflammatory syndrome induced by adjuvants (Shoenfeld's syndrome): clinical and immunological spectrum. Expert Rev Clin Immunol. 2013;9(4):361-73. doi:10. 1586/eci.13.2.

4. Esposito S, Prada E, Mastrolia MV, Tarantino G, Codeca C, Rigante D. Autoimmune/inflammatory syndrome induced by adjuvants (ASIA): clues and pitfalls in the pediatric background. Immunol Res. 2014;60(2-3):366-75. doi:10.1007/s12026-0148586-0.

5. Loke SC, Leow MK. Calcinosis cutis with siliconomas complicated by hypercalcemia. Endocr Pract. 2005;11(5):341-5. doi:10. 4158/EP.11.5.341.

6. Agmon-Levin N, Hughes GR, Shoenfeld Y. The spectrum of ASIA: 'autoimmune (Auto-inflammatory) syndrome induced by adjuvants'. Lupus. 2012;21(2):118-20. doi:10.1177/0961203311429316.

7. Israeli E, Agmon-Levin N, Blank M, Shoenfeld Y. Adjuvants and autoimmunity. Lupus. 2009;18(13):1217-25. doi:10.1177/ 0961203309345724. 
8. Pierangeli SS, Blank M, Liu X, Espinola R, Fridkin M, Ostertag $\mathrm{MV}$, et al. A peptide that shares similarity with bacterial antigens reverses thrombogenic properties of antiphospholipid antibodies in vivo. J Autoimmun. 2004;22(3):217-25. doi:10.1016/j.jaut. 2004.01.002.

9. Exley C, Siesjo P, Eriksson H. The immunobiology of aluminium adjuvants: how do they really work? Trends Immunol. 2010; 31(3):103-9. doi:10.1016/j.it.2009.12.009.

10. Marrack P, McKee AS, Munks MW. Towards an understanding of the adjuvant action of aluminium. Nat Rev Immunol. 2009;9(4):287-93. doi:10.1038/nri2510.

11. Eisenbarth SC, Colegio OR, O'Connor W, Sutterwala FS, Flavell RA. Crucial role for the $\mathrm{Nalp}_{3}$ inflammasome in the immunostimulatory properties of aluminium adjuvants. Nature. 2008; 453(7198):1122-6. doi:10.1038/nature06939.

12. Lidar M, Agmon-Levin N, Langevitz P, Shoenfeld Y. Silicone and scleroderma revisited. Lupus. 2012;21(2):121-7. doi:10. 1177/0961203311430703.

13. Caskey CI, Berg WA, Hamper UM, Sheth S, Chang BW, Anderson ND. Imaging spectrum of extracapsular silicone: correlation of US, MR imaging, mammographic, and histopathologic findings. Radiographics. 1999;19:S39-51. doi:10.1148/ radiographics.19.suppl_1.g99oc11s39.

14. Gherardi RK, Authier FJ. Macrophagic myofasciitis: characterization and pathophysiology. Lupus. 2012;21(2):184-9. doi:10. 1177/0961203311429557.

15. McCarthy J. Aeshetic breast surgery. Philadelphia: Saunders; 1990.

16. Sagi L, Baum S, Lyakhovitsky A, Barzilai A, Shpiro D, Trau H, et al. Silicone breast implant rupture presenting as bilateral leg nodules. Clin Exp Dermatol. 2009;34(5):e99-101. doi:10.1111/j. 1365-2230.2008.03196.x.

17. Wolfram D, Rabensteiner E, Grundtman C, Bock G, Mayerl C, Parson $\mathrm{W}$, et al. T regulatory cells and TH17 cells in peri-silicone implant capsular fibrosis. Plast Reconstr Surg. 2012;129(2):327e37e. doi:10.1097/PRS.0b013e31823aeacf.

18. Cohen Tervaert JW, Kappel RM. Silicone implant incompatibility syndrome (SIIS): a frequent cause of ASIA (Shoenfeld's syndrome). Immunol Res. 2013;56(2-3):293-8. doi:10.1007/ s12026-013-8401-3.

19. Gylbert L, Asplund O, Jurell G. Capsular contracture after breast reconstruction with silicone-gel and saline-filled implants: a 6-year follow-up. Plast Reconstr Surg. 1990;85(3):373-7.

20. Perricone C, Colafrancesco S, Mazor RD, Soriano A, AgmonLevin N, Shoenfeld Y. Autoimmune/inflammatory syndrome induced by adjuvants (ASIA) 2013: unveiling the pathogenic, clinical and diagnostic aspects. J Autoimmun. 2013;47:1-16. doi:10.1016/j.jaut.2013.10.004.

21. Goldblum RM, Pelley RP, O'Donell AA, Pyron D, Heggers JP. Antibodies to silicone elastomers and reactions to ventriculoperitoneal shunts. Lancet. 1992;340(8818):510-3.

22. Kaiser W, Biesenbach G, Stuby U, Grafinger P, Zazgornik J. Human adjuvant disease: remission of silicone induced autoimmune disease after explanation of breast augmentation. Ann Rheum Dis. 1990;49(11):937-8.

23. Caldeira M, Ferreira AC. Siliconosis: autoimmune/inflammatory syndrome induced by adjuvants (ASIA). Isr Med Assoc J. 2012;14(2):137-8.

24. Flassbeck D, Pfleiderer B, Klemens P, Heumann KG, Eltze E, Hirner AV. Determination of siloxanes, silicon, and platinum in tissues of women with silicone gel-filled implants. Anal Bioanal Chem. 2003;375(3):356-62. doi:10.1007/s00216-002-1694-z.

25. Barker DE, Retsky MI, Schultz S. "Bleeding" of silicone from bag-gel breast implants, and its clinical relation to fibrous capsule reaction. Plast Reconstr Surg. 1978;61(6):836-41.
26. Fenske TK, Davis P, Aaron SL. Human adjuvant disease revisited: a review of eleven post-augmentation mammoplasty patients. Clin Exp Rheumatol. 1994;12(5):477-81.

27. Tervaert JW, Stegeman CA, Kallenberg CG. Silicon exposure and vasculitis. Curr Opin Rheumatol. 1998;10(1):12-7.

28. Miyoshi K, Miyaoka G, Kobayashi Y, Itakura T, Higashihara M, Ono B. 2 Cases of hypergammaglobulinemia thought to be delayed sensitivity to added adjuvant in the human body. Arerugi. 1965;14:69-71.

29. Shoaib BO, Patten BM. Human adjuvant disease: presentation as a multiple sclerosis-like syndrome. South Med J. 1996;89(2): 179-88.

30. Nancy AL, Shoenfeld Y. Chronic fatigue syndrome with autoantibodies - the result of an augmented adjuvant effect of hepatitis-B vaccine and silicone implant. Autoimmun Rev. 2008;8(1):52-5. doi:10.1016/j.autrev.2008.07.026.

31. van Nunen SA, Gatenby PA, Basten A. Post-mammoplasty connective tissue disease. Arthritis Rheum. 1982;25(6):694-7.

32. Levy Y, Rotman-Pikielny P, Ehrenfeld M, Shoenfeld Y. Silicone breast implantation-induced scleroderma: description of four patients and a critical review of the literature. Lupus. 2009;18(13):1226-32. doi:10.1177/0961203309347795.

33. Bar-Meir E, Eherenfeld M, Shoenfeld Y. Silicone gel breast implants and connective tissue disease-a comprehensive review. Autoimmunity. 2003;36(4):193-7.

34. Kivity S, Katz M, Langevitz P, Eshed I, Olchovski D, Barzilai A. Autoimmune syndrome induced by adjuvants (ASIA) in the middle east: morphea following silicone implantation. Lupus. 2012;21(2):136-9. doi:10.1177/0961203311429551.

35. Parks CG, Conrad K, Cooper GS. Occupational exposure to crystalline silica and autoimmune disease. Environ Health Perspect. 1999;107(Suppl 5):793-802.

36. Hennekens $\mathrm{CH}$, Lee IM, Cook NR, Hebert PR, Karlson EW, LaMotte F, et al. Self-reported breast implants and connectivetissue diseases in female health professionals. A retrospective cohort study. JAMA. 1996;275(8):616-21.

37. Janowsky EC, Kupper LL, Hulka BS. Meta-analyses of the relation between silicone breast implants and the risk of connective-tissue diseases. N Engl J Med. 2000;342(11):781-90. doi:10.1056/NEJM200003163421105.

38. Sanchez-Guerrero J, Schur PH, Sergent JS, Liang MH. Silicone breast implants and rheumatic disease. Clinical, immunologic, and epidemiologic studies. Arthritis Rheum. 1994;37(2):158-68.

39. Gabriel SE, O'Fallon WM, Kurland LT, Beard CM, Woods JE, Melton LJ 3rd. Risk of connective-tissue diseases and other disorders after breast implantation. $\mathrm{N}$ Engl $\mathrm{J}$ Med. 1994;330(24):1697-702. doi:10.1056/NEJM199406163302401.

40. Bar-Meir E, Teuber SS, Lin HC, Alosacie I, Goddard G, Terybery $\mathrm{J}$, et al. Multiple autoantibodies in patients with silicone breast implants. J Autoimmun. 1995;8(2):267-77.

41. Zandman-Goddard G, Blank M, Ehrenfeld M, Gilburd B, Peter J, Shoenfeld Y. A comparison of autoantibody production in asymptomatic and symptomatic women with silicone breast implants. J Rheumatol. 1999;26(1):73-7.

42. Fryzek JP, Signorello LB, Hakelius L, Feltelius N, Ringberg A, Blot WJ, et al. Self-reported symptoms among women after cosmetic breast implant and breast reduction surgery. Plast Reconstr Surg. 2001;107(1):206-13.

43. Vera-Lastra O, Medina G, Cruz-Dominguez Mdel P, Ramirez P, Gayosso-Rivera JA, Anduaga-Dominguez $\mathrm{H}$, et al. Human adjuvant disease induced by foreign substances: a new model of ASIA (Shoenfeld's syndrome). Lupus. 2012;21(2):128-35. doi:10.1177/0961203311429317.

44. Vasey FB, Zarabadi SA, Seleznick M, Ricca L. Where there's smoke there's fire: the silicone breast implant controversy 
continues to flicker: a new disease that needs to be defined. J Rheumatol. 2003;30(10):2092-4.

45. Rahamim-Cohen D, Shoenfeld Y. The mosaic of autoimmunity. A classical case of inhalation of a polyclonal activating factor in a genetically and hormonally susceptible patient leading to multiple autoimmune diseases. Isr Med Assoc J. 2001;3(5):381-2.

46. Selmi C, Lu Q, Humble MC. Heritability versus the role of the environment in autoimmunity. J Autoimmun. 2012;39(4):249-52. doi:10.1016/j.jaut.2012.07.011.

47. Meier LG, Barthel HR, Seidl C. Development of polyarthritis after insertion of silicone breast implants followed by remission after implant removal in 2 HLA-identical sisters bearing rheumatoid arthritis susceptibility genes. J Rheumatol. 1997;24(9):1838-41.

48. Hajdu SD, Agmon-Levin N, Shoenfeld Y. Silicone and autoimmunity. Eur J Clin Invest. 2011;41(2):203-11. doi:10.1111/j. 1365-2362.2010.02389.x.

49. Colaris MJ, de Boer M, van der Hulst RR, Cohen Tervaert JW. Two hundreds cases of ASIA syndrome following silicone implants: a comparative study of 30 years and a review of current literature. Immunol Res. 2016;. doi:10.1007/s12026-016-8821-y.

50. Smack D, Norton SA, Fitzpatrick JE. Proposal for a pathogenesisbased classification of tumoral calcinosis. Int $\mathrm{J}$ Dermatol. 1996;35(4):265-71.

51. Tan AW, Ng HJ, Ang P, Goh YT. Extensive calcinosis cutis in relapsed acute lymphoblastic leukaemia. Ann Acad Med Singap. 2004;33(1):107-9.

52. Buchowski JM, Ahn NU, Ahn UM, McCarthy EF, Mehta MB. Disproportionately severe calcinosis cutis in an 88-year-old patient with CREST syndrome. Skeletal Radiol. 2001;30(8):478-81. doi:10.1007/s0025610300478.

53. Fuss M, Pepersack T, Gillet C, Karmali R, Corvilain J. Calcium and vitamin $\mathrm{D}$ metabolism in granulomatous diseases. Clin Rheumatol. 1992;11(1):28-36.

54. Barbour GL, Coburn JW, Slatopolsky E, Norman AW, Horst RL. Hypercalcemia in an anephric patient with sarcoidosis: evidence for extrarenal generation of 1,25-dihydroxyvitamin D. N Engl J Med. 1981;305(8):440-3. doi:10.1056/NEJM198108203050807.

55. Sharma OP. Hypercalcemia in granulomatous disorders: a clinical review. Curr Opin Pulm Med. 2000;6(5):442-7.

56. Krikorian A, Shah S, Wasman J. Parathyroid hormone-related protein: an unusual mechanism for hypercalcemia in sarcoidosis. Endocr Pract. 2011;17(4):e84-6. doi:10.4158/EP11060.CR.

57. Pinkston P, Saltini C, Muller-Quernheim J, Crystal RG. Corticosteroid therapy suppresses spontaneous interleukin 2 release and spontaneous proliferation of lung $\mathrm{T}$ lymphocytes of patients with active pulmonary sarcoidosis. J Immunol. 1987;139(3):755-60.

58. Kozeny GA, Barbato AL, Bansal VK, Vertuno LL, Hano JE. Hypercalcemia associated with silicone-induced granulomas.
N Engl J Med. 1984;311(17):1103-5. doi:10.1056/NEJM198410 253111707.

59. Visnyei K, Samuel M, Heacock L, Cortes JA. Hypercalcemia in a male-to-female transgender patient after body contouring injections: a case report. J Med Case Rep. 2014;8:71. doi:10.1186/ 1752-1947-8-71.

60. Camuzard O, Dumas P, Foissac R, Fernandez J, David S, Balaguer $\mathrm{T}$, et al. Severe granulomatous reaction associated with hypercalcemia occurring after silicone soft tissue augmentation of the buttocks: a case report. Aesthetic Plast Surg. 2014;38(1):95-9. doi:10.1007/s00266-013-0167-4.

61. Schanz J, Flux K, Kircher C, Tsioga M, Hartschuh W, Nawroth $\mathrm{PP}$, et al. Mirror, mirror on the wall: hypercalcemia as a consequence of modern cosmetic treatment with liquid silicone. Med Sci Monit. 2012;18(2):CS5-7.

62. Agrawal N, Altiner S, Mezitis NH, Helbig S. Silicone-induced granuloma after injection for cosmetic purposes: a rare entity of calcitriol-mediated hypercalcemia. Case Rep Med. 2013;2013: 807292. doi:10.1155/2013/807292.

63. Gyetko MR, Hsu CH, Wilkinson CC, Patel S, Young E. Monocyte 1 alpha-hydroxylase regulation: induction by inflammatory cytokines and suppression by dexamethasone and uremia toxin. J Leukoc Biol. 1993;54(1):17-22.

64. Rizzoli R, Feyen JH, Grau G, Wohlwend A, Sappino AP, Bonjour JP. Regulation of parathyroid hormone-related protein production in a human lung squamous cell carcinoma line. J Endocrinol. 1994;143(2):333-41.

65. Arin MJ, Bate J, Krieg T, Hunzelmann N. Silicone granuloma of the face treated with minocycline. J Am Acad Dermatol. 2005;52(2 Suppl 1):53-6. doi:10.1016/j.jaad.2004.07.014.

66. Barre PE, Gascon-Barre M, Meakins JL, Goltzman D. Hydroxychloroquine treatment of hypercalcemia in a patient with sarcoidosis undergoing hemodialysis. Am J Med. 1987;82(6): 1259-62.

67. Agrawal V, Crisi GM, D’Agati VD, Freda BJ. Renal sarcoidosis presenting as acute kidney injury with granulomatous interstitial nephritis and vasculitis. Am J Kidney Dis. 2012;59(2):303-8. doi:10.1053/j.ajkd.2011.09.025.

68. Ponce C, Gujral JS. Renal failure and hypercalcemia as initial manifestations of extrapulmonary sarcoidosis. South Med J. 2004;97(6):590-2.

69. Jara LJ, Garcia-Collinot G, Medina G, Cruz-Dominguez MD, Vera-Lastra O, Carranza-Muleiro RA, et al. Severe manifestations of autoimmune syndrome induced by adjuvants (Shoenfeld's syndrome). Immunol Res. 2016;. doi:10.1007/s12026-0168811-0. 